\title{
Indicadores de qualidade de Latossolo relacionados à degradação de pastagens
}

\author{
Fabrício Marinho Lisbôa(1), Guilherme Kangussu Donagemma ${ }^{(2)}$, Diego Lang Burak ${ }^{(3)}$, \\ Renato Ribeiro Passos ${ }^{(3)}$ e Eduardo de Sá Mendonça( ${ }^{(3)}$
}

\begin{abstract}
(1)Universidade Estadual do Norte Fluminense Darcy Ribeiro, Avenida Alberto Lamego, no 2.000, Parque Califórnia, CEP 28013-602 Campos dos Goytacazes, RJ, Brasil. E-mail: fabriciomarinho87@yahoo.com ${ }^{(2)}$ Embrapa Solos, Rua Jardim Botânico, no 1.024, Jardim Botânico, CEP 22460-000 Rio de Janeiro, RJ, Brasil. E-mail: guilherme.donagemma@embrapa.br ${ }^{(3)}$ Universidade Federal do Espírito Santo, Alto Universitário, s/no, Guararema, CEP 29500-000 Alegre, ES, Brasil. E-mail: dlburak@hotmail.com, renatoribeiropassos@hotmail.com, esmjplia@gmail.com
\end{abstract}

Resumo - O objetivo deste trabalho foi selecionar os indicadores de qualidade de solo mais relacionados à degradação de pastagens, e estabelecer os limites de diferentes níveis de degradação a partir desses indicadores. Cinco pastagens sobre Latossolo Vermelho-Amarelo foram selecionadas, em terço médio da encosta, com diferentes níveis visuais de degradação, tendo-se considerado as variáveis: perda de vigor, presença de espécies espontâneas, solo exposto e erosão. Avaliou-se a cobertura do solo, e coletaram-se amostras nas profundidades de $0,00-0,05,0,05-0,10$ e $0,10-0,20 \mathrm{~m}$, para as análises físicas, químicas e de matéria orgânica. Diferentemente da classificação visual, a análise de agrupamento das variáveis de cobertura indicou apenas dois níveis de degradação: de baixo a moderado e de alto a muito alto, com percentual de classificações corretas acima de $87 \%$ na análise discriminante, em que o solo exposto foi a variável mais assertiva para discriminar os níveis de degradação. A matéria orgânica leve e o componente $\mathrm{Ca}+\mathrm{Mg}$ foram os indicadores de qualidade mais relacionados aos índices de degradação de pastagens à profundidade de $0,00-0,05 \mathrm{~m}$; enquanto o teor de potássio foi o indicador mais relacionado aos índices às profundidades de $0,05-0,10$ e $0,10-0,20 \mathrm{~m}$.

Termos para indexação: Urochloa, cobertura de solo, degradação do solo, matéria orgânica leve.

\section{Oxisol quality indicators related to degradation of pastures}

\begin{abstract}
The objective of this work was to select the soil quality indicators more related to pasture degradation and to establish the limits of the different degradation levels using these indicators. Five pasture areas on an Oxisol were selected in the medium third of the slope, with different levels of visual degradation, considering the following variables: loss of vigor, presence of weeds, exposed bare soil, and erosion. Soil cover was evaluated, and soil samples were collected at the $0.00-0.05,0.05-0.10$, and $0.10-0.20-\mathrm{m}$ soil depths, for physical, chemical, and organic matter analyses. Differently from the visual classification, the cluster analysis of the cover variables only indicated two degradation levels: low to moderate and high to very high, with classification percentage above $87 \%$ in the discriminant analysis, in which bare soil was the most assertive variable for discriminating the degradation levels. Light organic matter and the $\mathrm{Ca}+\mathrm{Mg}$ component were the indicators most related to pasture degradation indices at $0.00-0.05 \mathrm{~m}$; while potassium content was the indicator most related to those indices at the $0.05-0.10$ and $0.10-0.20-\mathrm{m}$ soil depths.
\end{abstract}

Index terms: Urochloa, vegetation cover, soil degradation, light organic matter.

\section{Introdução}

As pastagens extensivas no Brasil são, em sua maioria, implementadas com gramíneas do gênero Urochloa (syn. Brachiaria), que apresentam elevada rusticidade, produção de matéria seca e adaptação, além de bom valor nutritivo (Costa et al., 2005). No entanto, sua boa adaptabilidade a ambientes de clima tropical não impede que se degradem com práticas de manejo inadequadas, baixa fertilidade do solo e adversidades climáticas. No Estado do Espírito
Santo, estimou-se que, de 1,32 milhão de hectares de pastagens, aproximadamente 239 mil hectares $(18,10 \%)$ estariam sob alguma forma de degradação (Barreto \& Sartori, 2012), o que justifica a necessidade de monitoramento dos níveis atuais de degradação nessas áreas, para servir de base para políticas de manejos mais sustentáveis.

Segundo Spain \& Gualdrón (1988), uma pastagem pode ser considerada degradada quando sofre diminuição considerável de sua produção, sob 
determinadas condições edafoclimáticas e bióticas. Os autores propuseram uma classificação visual para a avaliação da degradação das pastagens em níveis de 1 a 5, descritos a seguir. O nível 1 (leve) representa a perda de vigor e a qualidade abaixo de $25 \%$. O nível 2 (moderado) considera o nível 1 com a população da forrageira principal entre 25 a $50 \%$. O nível 3 (forte) considera os níveis 1 e 2 com os aparecimentos de espécies espontâneas entre 50 e $75 \%$. O nível 4 (muito forte) considera os níveis 1,2 e 3 e o número de formigueiros/termiteiros maior que $75 \%$. O nível 5 (muito forte) considera os níveis 1, 2, 3 e 4 acrescido do nível de solo exposto maior que $75 \%$. O nível 6 (muito forte) considera a junção de todos os níveis acrescida da erosão severa do solo.

Apesar de as avaliações visuais terem destaques recentes na literatura, como forma de estabelecer níveis de degradação (Albernaz \& Lima, 2007; Mellone et al., 2008; Niero et al., 2010), a degradação das pastagens pode ser mais bem retratada por meio de alterações nos indicadores de qualidade do solo (Moreira et al., 2005; Santos et al., 2009a; Pessoa et al., 2012). De acordo com Costa et al. (2012), indicadores físicos, como elevados valores de resistência à penetração, são eficientes em discriminar áreas com menor crescimento e produção de raízes, que reduzem a capacidade de absorção do sistema radicular e afetam a produção de biomassa aérea. Assim, ante a subjetividade das avaliações visuais, uma maior eficácia no monitoramento de níveis de degradação de pastagens pode ser alcançada a partir de indicadores de qualidade do solo que reflitam a diminuição na produção de biomassa.

Indicadores de qualidade do solo são propriedades mensuráveis que indicam a capacidade do solo de sustentar e promover a qualidade ambiental, e podem ser divididos em químicos, físicos e biológicos (Murphy et al., 2006). Rocha Junior et al. (2014) trabalharam em áreas de mata, capoeira e pastagens, sobre Argissolo Vermelho, e concluíram que a matéria orgânica particulada e a matéria orgânica dissolvida são os indicadores mais sensíveis para diferenciação dos níveis de degradação dessas áreas. Marchão et al. (2007) destacaram a retenção de água como indicadora da qualidade físico-hídrica de um Latossolo Vermelho, manejado com sistema de integração lavoura-pecuária. Essa técnica, entretanto, pode ter uso limitado, pois depende de centrífuga para sua determinação.
Ressalta-se que há necessidade da integração de um conjunto de indicadores (visual e solo) com maior poder discriminatório, para a distinção de níveis de degradação de pastagens, uma vez que o uso de diferentes indicadores irá fornecer informações do solo como todo (Murphy et al., 2006) e, ainda, buscar indicadores, na medida do possível, de fácil determinação ou execução, fáceis de serem obtidos e de baixo custo. Esses indicadores podem variar com o tipo de solo, manejo, clima e, consequentemente, com as especificidades de cada região.

O objetivo deste trabalho foi selecionar os indicadores de qualidade de solo mais relacionados à degradação de pastagens, e estabelecer os limites de diferentes níveis de degradação a partir desses indicadores.

\section{Material e Métodos}

O estudo foi realizado na Bacia do Rio Alegre, no Município de Alegre, ES, entre 231005 e 228746 m E, e 7689194 e $7688202 \mathrm{~m} \mathrm{~N}$, pelo sistema UTM (Datum SAD 69, zona 24S). O clima, segundo classificação de Köppen-Geiger, é do tipo Cwa: chuvoso no verão e seco no inverno (Santos et al., 2009b). A precipitação acumulada anual é de $1.630 \mathrm{~mm}$, com temperatura mínima de $18,85^{\circ} \mathrm{C}$ e máxima de $29,93^{\circ} \mathrm{C}$, obtidos na Estação Meteorológica de Alegre (Incaper..., 2014).

Cinco áreas de pastagens, em condições pedoclimáticas similares, foram selecionadas. Essas pastagens encontravam-se sobre Latossolo Vermelho-Amarelo distrófico argiloso, conforme classificação de Santos et al. (2013), com face de exposição ao sol Oeste/Norte, pedoforma convexaconvexa, altitude de 635 e $673 \mathrm{~m}$ e declividade de 12 a $32 \%$. Isto está em conformidade com Trasar-Cepeda et al. (1998), que recomendam condições pedogenéticas homogêneas para o desenvolvimento de índices de qualidade, pois a variabilidade dos atributos do solo em paisagens mais acidentadas dificulta a avaliação mais criteriosa da relação entre esses indicadores e os distúrbios em razão do manejo.

A avaliação visual das pastagens foi realizada em janeiro de 2013, tendo-se seguido os preceitos de Spain \& Gualdrón (1988) e estabelecido cinco níveis de degradação associados ao histórico de uso das áreas (Tabela 1). As cinco áreas de pastagem apresentavam o mesmo gênero forrageiro, com predomínio de 
Urochloa decumbens. As práticas de manejo adotadas foram as roçadas, as queimadas esporádicas e os ajustes da taxa de lotação, conforme a disponibilidade de forragem, sem calagem e nem adubação. As principais características químicas e físicas do solo das áreas utilizadas nesse estudo encontram-se na Tabela 2.

Entre as espécies espontâneas de maior ocorrência, observaram-se a mata-pasto (Eupatorium sp.), o carrapicho (Cenchrus sp.) e o capim-bermuda (Cynodon $\mathrm{sp}$.), que foram reunidas em espécies espontâneas de folha estreita (monocotiledôneas, como carrapicho e capim-bermuda) e espécies espontâneas de folha larga (dicotiledôneas, como mata-pasto).

A taxa de cobertura do solo foi determinada pelo método da trena, conforme adaptação do método da corda (Costa et al., 2000) realizado por Rocha Junior et al. (2014). Este método consiste no uso de uma trena com $50 \mathrm{~m}$ de comprimento, estendida no sentido das curvas de nível. Realizaram-se as seguintes observações (a cada metro): presença de Urochloa; solo exposto; e a presença de espécies espontâneas de folha larga e espécies espontâneas de folha estreita, tendo-se determinado o percentual de cada espécie em relação ao número total de pontos. Ao final da observação, a trena foi estendida novamente a um metro acima da posição anterior, e novas observações foram realizadas, tendo-se repetido este procedimento 10 vezes, no total de 500 pontos por área, com área total de $500 \mathrm{~m}^{2}$ por pastagem.

No interior da área observada de $500 \mathrm{~m}^{2}$, de cada pastagem, abriram-se quatro minitrincheiras, de forma aleatória, e coletaram-se amostras deformadas e indeformadas (anéis) e blocos de $5 \times 5 \times 5 \mathrm{~cm}$, de três profundidades: $0,00-0,05,0,05-0,10$ e $0,10-0,20 \mathrm{~m}$. Com a finalidade de se obter parâmetros comparativos entre as pastagens e um ambiente natural, também foram coletadas amostras de solo de uma área de mata secundária, em regeneração, próxima das áreas de pastagem.

As amostras deformadas foram passadas em peneira de malha de $2 \mathrm{~mm}$, após secagem ao ar, tendo-se assim obtido a terra fina seca ao ar (TFSA). As amostras indeformadas foram coletadas em anel volumétrico, com auxílio do amostrador de Uhland. Os blocos foram destorroados manualmente, passados em peneira de malha de $4 \mathrm{~mm}$ e retidos em peneira de $2 \mathrm{~mm}$, para obtenção dos agregados para a avaliação do estado de agregação dos solos.

Nas amostras de TFSA, foram determinadas as seguintes características do solo: $\mathrm{pH}$ em água; $\mathrm{Al}^{3+}$, $\mathrm{Ca}^{2+}, \mathrm{Mg}^{2+}$ e $\mathrm{Na}^{+}$trocáveis, extraídos com $\mathrm{KCl}$ a $1 \mathrm{~mol} \mathrm{~L}^{-1}$, à proporção $1: 10 ; \mathrm{Al}^{3+}$, por titulação com $\mathrm{NaOH}$ a $0,025 \mathrm{~mol} \mathrm{~L}^{-1} ; \mathrm{Ca}^{2+}$ e $\mathrm{Mg}^{2+}$, por espectrofotometria de absorção atômica; $\mathrm{Na}^{+}$, por fotometria de chama; $\mathrm{K}$ e $\mathrm{P}$ disponíveis, por extração com Mehlich-1 ( $\mathrm{HCl} 0,05 \mathrm{~mol} \mathrm{~L}^{-1}+\mathrm{H}_{2} \mathrm{SO}_{4} 0,0125 \mathrm{~mol}$ $\left.\mathrm{L}^{-1}\right)$, à proporção $1: 10$; e $\mathrm{H}+\mathrm{Al}$, por $\mathrm{Ca}(\mathrm{OAc})_{2}$ a 0,5 mol L ${ }^{-1}, c o m ~ p H ~ 7,0$ ajustado, à proporção 1:15, titulado com $\mathrm{NaOH}$ a $0,0606 \mathrm{~mol} \mathrm{~L}^{-1}$ (Donagema et al., 2011). $\mathrm{O}$ fósforo remanescente foi determinado a partir de 5 $\mathrm{cm}^{3}$ de TFSA, com $50 \mathrm{~mL}$ de solução de $\mathrm{CaCl}_{2}$ a 10 mmol L-1 , que continha $60 \mathrm{mg} \mathrm{L}^{-1}$ de $\mathrm{P}$ (Alvarez V et al., 2000). Foram determinados, ainda, o carbono orgânico total (COT), por oxidação via úmida; o carbono solúvel (Csol), em água; a matéria orgânica leve (MOL), em água; a atividade da biomassa microbiana, a partir do

Tabela 1. Descrição dos níveis de degradação das pastagens e histórico de uso.

\begin{tabular}{lcccccc}
\hline Pastagem & Coordenada & $\begin{array}{c}\text { Idade } \\
(\text { anos })\end{array}$ & $\begin{array}{c}\text { Animais } \\
\left(\mathrm{UA} \mathrm{ha}^{-1}\right)\end{array}$ & $\begin{array}{c}\text { Histórico } \\
\text { da área }^{(1)}\end{array}$ & $\begin{array}{c}\text { Nescrição da pastagem de } \\
\text { degradação }^{(3)}\end{array}$ \\
\hline P1 & $\begin{array}{c}20^{\circ} 53,210^{\prime} \mathrm{S} \\
41^{\circ} 36,446^{\prime} \mathrm{W}\end{array}$ & 3 & 1,6 & Mata/café/pasto & Alto vigor e qualidade, massa verde abundante. & Leve \\
\hline P2 & $\begin{array}{c}20^{\circ} 52,726^{\prime} \mathrm{S} \\
41^{\circ} 35,235^{\prime} \mathrm{W}\end{array}$ & 12 & 2,6 & Mata/café/pasto & Vigor e qualidade, massa verde menos abundante. & Moderado \\
\hline P3 & $\begin{array}{c}20^{\circ} 53,123^{\prime} \mathrm{S} \\
41^{\circ} 36,345^{\prime} \mathrm{W}\end{array}$ & 15 & 1,8 & Mata/café/pasto & $\begin{array}{c}\text { Sem vigor, pouca massa verde e presença de algumas espécies } \\
\text { espontâneas. }\end{array}$ & Forte \\
\hline P4 & $\begin{array}{c}20^{\circ} 52,780^{\prime} \mathrm{S} \\
41^{\circ} 35,168^{\prime} \mathrm{W}\end{array}$ & 12 & 2,6 & Mata/café/pasto & $\begin{array}{c}\text { Sem vigor, pouca massa verde, presença maior de espécies } \\
\text { espontâneas, solo exposto e presença de formigueiro. }\end{array}$ & Muito forte \\
\hline P5 & $20^{\circ} 52,770^{\prime} \mathrm{S}$ & 7 & 2 & Mata/café/pasto & $\begin{array}{c}\text { Sem vigor, pouca massa verde, presença de espécies espontâneas, } \\
\text { solo exposto, voçorocas, e presença de formigueiros e termiteiros. }\end{array}$ Extremo \\
\hline
\end{tabular}

${ }^{(1)}$ Conforme informações obtidas com os proprietários. ${ }^{(2)}$ Estabelecidos segundo Spain \& Gualdrón (1988). ${ }^{(3)}$ Observados visualmente. 
$\mathrm{CO}_{2}$ liberado; e o $\mathrm{C}$ da biomassa microbiana (CBM), pelo método da irradiação-extração (Mendonça \& Matos, 2005).

Tabela 2. Média dos principais atributos químicos e físicos nas camadas de solo das áreas estudadas.

\begin{tabular}{|c|c|c|c|c|c|c|}
\hline \multirow[t]{2}{*}{ Atributo } & \multirow[t]{2}{*}{ Mata } & \multicolumn{5}{|c|}{ Níveis de degradação das pastagens $^{(1)}$} \\
\hline & & Leve & Moderado & Forte & $\begin{array}{c}\text { Muito } \\
\text { forte }\end{array}$ & Extremo \\
\hline & \multicolumn{6}{|c|}{$0,00-0,05 \mathrm{~m}$} \\
\hline COT & 1,58 & 1,71 & 1,30 & 1,74 & 1,54 & 1,61 \\
\hline $\mathrm{pH}$ & 4,67 & 5,25 & 5,19 & 5,09 & 4,82 & 5,05 \\
\hline $\mathrm{P}$ & 3,12 & 2,96 & 2,12 & 2,90 & 2,04 & 2,89 \\
\hline K & 54,25 & 42,00 & 65,5 & 30,5 & 27,00 & 36,00 \\
\hline $\mathrm{Ca}+\mathrm{Mg}$ & 0,22 & 0,90 & 0,34 & 0,18 & 0,07 & 0,21 \\
\hline $\mathrm{Al}$ & 0,85 & 0,35 & 0,16 & 0,84 & 0,64 & 0,71 \\
\hline $\mathrm{H}+\mathrm{Al}$ & 7,63 & 7,65 & 6,21 & 8,75 & 7,24 & 7,14 \\
\hline DS & 1,22 & 1,16 & 1,43 & 1,25 & 1,22 & 1,47 \\
\hline Pmacro & 0,19 & 0,14 & 0,14 & 0,15 & 0,16 & 0,15 \\
\hline \multirow[t]{2}{*}{ Pmicro } & 0,36 & 0,42 & 0,32 & 0,36 & 0,39 & 0,32 \\
\hline & \multicolumn{6}{|c|}{$0,05-0,10 \mathrm{~m}$} \\
\hline COT & 1,35 & 1,65 & 1,13 & 1,63 & 1,50 & 1,50 \\
\hline $\mathrm{pH}$ & 4,59 & 5,21 & 5,15 & 5,18 & 4,67 & 5,00 \\
\hline $\mathrm{P}$ & 2,80 & 2,52 & 2,05 & 2,17 & 2,04 & 2,70 \\
\hline K & 51,33 & 41,25 & 37,33 & 12,33 & 19,00 & 29,65 \\
\hline $\mathrm{Ca}+\mathrm{Mg}$ & 0,06 & 0,38 & 0,19 & 0,14 & 0,09 & 0,11 \\
\hline $\mathrm{Al}$ & 0,83 & 0,64 & 0,35 & 0,81 & 0,90 & 0,99 \\
\hline $\mathrm{H}+\mathrm{Al}$ & 7,47 & 8,33 & 6,41 & 8,75 & 7,18 & 7,78 \\
\hline DS & 1,20 & 1,16 & 1,38 & 1,18 & 1,23 & 1,37 \\
\hline Pmacro & 0,25 & 0,16 & 0,18 & 0,19 & 0,15 & 0,18 \\
\hline \multirow[t]{2}{*}{ Pmicro } & 0,31 & 0,40 & 0,32 & 0,34 & 0,38 & 0,33 \\
\hline & \multicolumn{6}{|c|}{$0,10-0,20 \mathrm{~m}$} \\
\hline COT & 1,31 & 1,44 & 1,00 & 1,54 & 1,39 & 1,40 \\
\hline $\mathrm{pH}$ & 4,53 & 5,12 & 5,28 & 5,14 & 4,87 & 4,95 \\
\hline $\mathrm{P}$ & 2,52 & 2,29 & 1,74 & 2,33 & 1,81 & 2,51 \\
\hline K & 37,25 & 36,00 & 37,75 & 9,75 & 13,75 & 24,00 \\
\hline $\mathrm{Ca}+\mathrm{Mg}$ & 0,02 & 0,18 & 0,22 & 0,15 & 0,08 & 0,30 \\
\hline $\mathrm{Al}$ & 0,78 & 0,66 & 0,35 & 0,71 & 0,91 & 1,20 \\
\hline $\mathrm{H}+\mathrm{Al}$ & 7,47 & 8,19 & 5,69 & 8,39 & 6,89 & 8,11 \\
\hline DS & 1,15 & 1,09 & 1,33 & 1,09 & 1,18 & 1,34 \\
\hline Pmacro & 0,24 & 0,18 & 0,17 & 0,22 & 0,18 & 0,21 \\
\hline Pmicro & 0,33 & 0,40 & 0,35 & 0,36 & 0,38 & 0,32 \\
\hline
\end{tabular}

${ }^{(1)}$ Ver descrição na Tabela 1. COT, carbono orgânico total, dag $\mathrm{kg}^{-1} ; \mathrm{pH}$ em $\mathrm{H}_{2} \mathrm{O} ; \mathrm{P}, \mathrm{mg} \mathrm{dm}^{-3} ; \mathrm{K}, \mathrm{mg} \mathrm{dm}^{-3} ; \mathrm{Ca}+\mathrm{Mg}, \mathrm{cmol}_{\mathrm{c}} \mathrm{dm}^{-3} ; \mathrm{Al}, \mathrm{cmol}_{\mathrm{c}} \mathrm{dm}^{-3} ; \mathrm{H}+\mathrm{Al}$, acidez potencial, $\mathrm{cmol}_{\mathrm{c}} \mathrm{dm}^{-3}$; Ds, densidade do solo, $\mathrm{kg} \mathrm{dm}^{-3}$; Pmacro, macroporosidade, $\mathrm{m} \mathrm{m}^{-3}$; Pmicro, microporosidade, $\mathrm{m} \mathrm{m}^{-3}$.
A argila dispersa em água (ADA) foi determinada com $10 \mathrm{~g}$ de TFSA e $200 \mathrm{~mL}$ de água, em agitador tipo Wagner (Donagema et al., 2011). A densidade do solo (Ds) foi obtida pelo método do anel volumétrico, e a densidade de partículas (Dp), pelo método do balão volumétrico. A partir dos valores de Ds e Dp, calcularam-se os valores de porosidade total (Ptotal) (Donagema et al., 2011). A microporosidade (Pmicro) foi determinada em mesa de tensão, com $60 \mathrm{~cm}$ de coluna de água, e a macroporosidade (Pmacro) foi obtida pela equação Pmacro $=$ Ptotal - Pmicro. A análise de estabilidade de agregados foi obtida por peneiramento via úmida (Donagema et al., 2011). $O$ índice de estabilidade de agregados (IEA) foi calculado conforme Lacerda et al. (2005).

Os dados foram testados quanto à normalidade (Shapiro \& Wilk, 1965) e transformados, quando necessário, tendo-se optado pelas transformações que apresentaram distribuição normal. A fim de testar os níveis de degradação observados visualmente, aplicou-se a análise de agrupamento com as variáveis de cobertura vegetal. Quatro métodos de aglomeração foram utilizados: ligação simples, ligação completa, UPGMA e Ward, a partir da distância euclidiana como medida de dissimilaridade. Optou-se pelo método que melhor distinguiu os níveis de degradação. Em seguida, a análise discriminante canônica foi aplicada, com os novos níveis de degradação como variáveis dependentes, e os percentuais dos atributos vegetais como variáveis independentes, o que gerou as funções discriminantes e o percentual de classificações correta, por meio do atributos de cobertura.

Os indicadores do solo de maior importância para a distinção dos níveis de degradação das pastagens foram selecionados a partir de análise discriminante, tendo-se utilizado o método de inclusão progressiva passo a passo ("forward stepwise"). Para isso, os níveis obtidos após a análise de agrupamento foram utilizados novamente como variáveis dependentes, e os indicadores do solo como variáveis independentes. Utilizou-se o critério lambda de seleção de Wilks, com significância de 0,15 . Por esse procedimento, também foram geradas as funções discriminantes e o percentual de classificações corretas, por meio dos indicadores do solo.

A análise de variância foi realizada entre os níveis de degradação e mata, com os indicadores do solo selecionado pelo método "forward stepwise". Todos os 
procedimentos estatísticos descritos foram realizados por meio do programa $\mathrm{R}$.

\section{Resultados e Discussão}

Os cinco níveis de degradação das pastagens, observados visualmente no início do estudo e utilizados como variáveis dependentes na análise discriminante (AD), possibilitaram $60 \%$ de classificações corretas. Por esse motivo, optou-se por realizar a análise de agrupamento, que forneceu dois novos níveis (Figura 1), utilizados em uma nova análise discriminante (Tabela 3). Os dois novos níveis foram denominados, a partir de então, como: baixo a moderado (composto pelos níveis leve, moderado e extremo); e alto a muito alto (composto pelos níveis forte e muito forte). A utilização dos novos níveis de degradação aumentou para $87 \%$ o número de

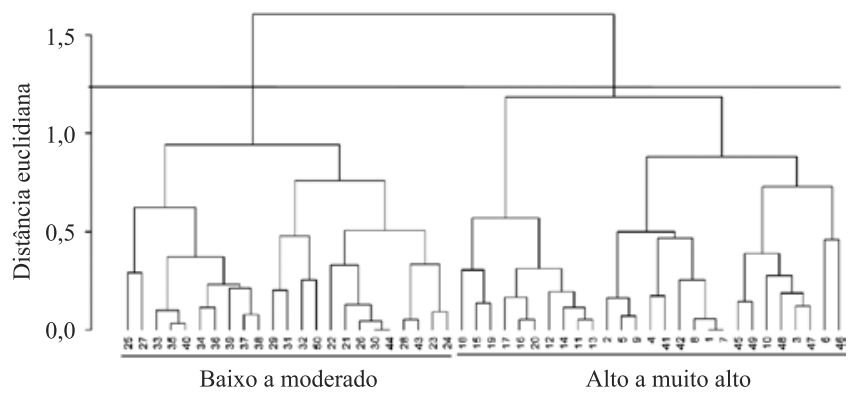

Figura 1. Agrupamento formado partir da distância euclidiana, pelo método de ligação completa dos níveis de degradação das pastagens em Alegre, ES. classificações corretas pela análise discriminante, superiores aos $73 \%$ alcançados por McInnes et al. (2015). Isso deixa clara a subjetividade do método proposto por Spain \& Gualdrón (1988) e que técnicas de levantamento da cobertura vegetal, como o método da trena, utilizado no presente estudo, podem ser usadas para maior precisão do estabelecimento de níveis de degradação das pastagens.

A percentagem de erro de $13 \%$, no nível alto a muito alto de degradação, provavelmente resultou da ambiguidade de plantas de ocorrência espontânea com padrão visual semelhante à forrageira principal, o que dificultou a distinção dos níveis de degradação (McInnes et al., 2005; Andrade et al., 2013). Esse nível de degradação também apresentou o menor percentual de Urochloa, além de maior percentual de solo exposto (Tabela 4). No entanto, o nível baixo a moderado de degradação constituiu-se das pastagens com maior percentagem de Urochloa.

No resultado da análise discriminante (Tabela 3), a maior carga discriminante foi atribuída ao solo exposto $(-0,80)$, Urochloa $(0,78)$ e vegetação espontânea de folhas largas $(-0,77)$, que contribuíram de forma significativa para a diferenciação dos níveis de degradação pela função discriminante, o que indica que as avaliações visuais devem dar maior importância a essas variáveis. A presença de vegetação espontânea de folha estreita não contribuiu expressivamente para essa diferenciação $(0,57)$.

A partir dos resultados, sugerem-se os seguintes níveis modificados de Spain \& Gualdrón (1988): nível baixo a moderado de degradação, que apresenta

Tabela 3. Parâmetros de ajuste da função discriminante dos cinco níveis visuais de degradação, quanto à cobertura do solo, bem como cargas discriminantes das variáveis utilizadas e capacidade preditiva da função discriminante.

\begin{tabular}{|c|c|c|c|c|c|}
\hline \multirow[t]{2}{*}{ Variável } & \multicolumn{2}{|c|}{ Função discriminante } & \multicolumn{3}{|c|}{ Capacidade preditiva da função discriminante dos atributos de cobertura do solo } \\
\hline & Ajuste do modelo & Carga discriminante & Baixo a moderado & Alto a moderado & Classificação correta (\%) \\
\hline Proporção explicada & 1,00 & - & - & - & - \\
\hline Autovalor & 2,72 & - & - & - & - \\
\hline Correlação canônica & 0,85 & - & - & - & - \\
\hline Urochloa & - & 0,78 & - & - & - \\
\hline Solo exposto & - & $-0,80$ & - & - & - \\
\hline Folhas estreitas & - & 0,57 & - & - & - \\
\hline Folhas largas & - & $-0,77$ & - & - & - \\
\hline \multicolumn{6}{|l|}{ Nível de degradação } \\
\hline Baixo a moderado & - & - & 100 & 0 & 100 \\
\hline Alto a muito alto & - & - & 13 & 87 & 87 \\
\hline
\end{tabular}


pastagem com massa verde de abundante a pouco abundante e presença pouco abundante de invasoras de folha larga; nível alto a muito alto de degradação, que apresenta massa verde muito pouco abundante e presença abundante de invasoras de folha larga, com presença de pouco a muito solo exposto, presença de formigueiros e termiteiros, além de voçorocas.

Os atributos do solo, utilizados para a seleção como indicadores dos níveis de degradação, estão apresentados na Tabela 5. Nos diferentes níveis de pastagens degradadas, o Latossolo Vermelho-Amarelo apresentou as seguintes características: níveis baixos de fertilidade, para todos os macronutrientes; acidez média, quanto aos valores de $\mathrm{pH}$; teores médios de alumínio; e teores elevados de acidez potencial (Prezotti et al., 2007). Estes resultados estão dentro do esperado para os Latossolos, que apresentam baixa fertilidade em razão de seu material de origem, elevado intemperismo e remoção de $\mathrm{SiO}_{2}$ e acúmulo de $\mathrm{Al}_{2} \mathrm{O}_{3}$ em sua fase mineral, com predomínio de caulinita e óxidos de ferro e alumínio (Araujo-Junior et al., 2011). Além disso, o solo selecionado está relacionado a um manejo que não se faz calagem nem adubação.

A densidade do solo (DS) apresentou média acima do encontrado na área de mata próxima, e os maiores valores encontrados, nas três profundidades $(0,00$ $0,05,0,05-0,10$ e $0,10-0,20 \mathrm{~m})$, foram $1,22,1,20$ e 1,15 $\mathrm{kg} \mathrm{dm}^{-3}$, respectivamente. Nas pastagens, a DS diminuiu com a profundidade, o que pode estar relacionado ao tráfego de animais que pode provocar aumento da DS em superfície, sobretudo na camada de $0,00-0,05 \mathrm{~m}$ de profundidade. As maiores densidades do solo podem resultar em menores valores de macroporosidade (Michelon et al., 2009); isto pôde ser observado na área de mata, que apresentou maiores valores para esse

Tabela 4. Média \pm desvio-padrão das variáveis de cobertura e dos níveis de degradação das pastagens, após a análise de agrupamento.

\begin{tabular}{lcc}
\hline Cobertura & Baixo a moderado & Alto a muito Alto \\
\hline Urochloa & $81,11 \pm 11,77$ & $57,04 \pm 15,25$ \\
Solo exposto & $9,56 \pm 5,56$ & $23,39 \pm 9,21$ \\
EFE & $7,85 \pm 8,84$ & $0,52 \pm 1.24$ \\
EFL & $1,48 \pm 2,52$ & $19,04 \pm 14,70$ \\
\hline
\end{tabular}

EFE, planta espontânea de folha estreita; e EFL, planta espontânea de folha larga. atributo, nas três profundidades $(0,19,0,25$ e $0,24 \mathrm{~m}$ $\mathrm{m}^{-3}$, respectivamente), em comparação às pastagens.

Para a seleção de indicadores de qualidade do solo com maior relação com os níveis de degradação, utilizou-se a análise discriminante, com seleção de variáveis pelo método forward stepwise. Em uma primeira etapa, selecionaram-se os atributos de cada profundidade, promissores para a separação dos níveis de degradação, para, posteriormente incluí-los dentro do conjunto de atributos com todas as profundidades (Tabela 6).

À profundidade $0,00-0,05 \mathrm{~m}$ (Tabela 6), a percentagem geral de erro foi menor do que à profundidade $0,10-0,20 \mathrm{~m}$, o que indica que os atributos selecionados em superfície (MOL, $\mathrm{H}+\mathrm{Al}$, COT, DS, $\mathrm{Ca}+\mathrm{Mg}$ ) apresentam uma melhor relação com os níveis de degradação. A camada superficial do solo em sistemas pastoris pode ser mais sensível às mudanças de manejo, principalmente pela compactação

Tabela 5. Média \pm desvio-padrão dos atributos, medidos em três profundidades do solo sob pastagens, usados para a seleção de indicadores.

\begin{tabular}{|c|c|c|c|}
\hline \multirow[t]{2}{*}{ Atributo } & \multicolumn{3}{|c|}{ Profundidade do solo } \\
\hline & $0,00-0,05 \mathrm{~m}$ & $0,05-0,10 \mathrm{~m}$ & $0,10-0,20 \mathrm{~m}$ \\
\hline $\mathrm{AM}\left(\mathrm{mg} \mathrm{C}-\mathrm{CO}_{2} 100 \mathrm{~cm}^{-3}\right)$ & $47,4 \pm 13,1$ & $42,4 \pm 14,2$ & $46,0 \pm 14,2$ \\
\hline $\operatorname{CBM}\left(\mu \mathrm{g} \mathrm{g}^{-1}\right)$ & $1,203 \pm 1,167$ & $947 \pm 995$ & $1,241 \pm 1,234$ \\
\hline $\operatorname{COT}\left(\mathrm{g} \mathrm{kg}^{-1}\right)$ & $15,8 \pm 2,1$ & $14,8 \pm 2,1$ & $13,6 \pm 2,1$ \\
\hline $\operatorname{MOL}\left(\mathrm{g} \mathrm{kg}^{-1}\right)$ & $3,23 \pm 1,44$ & $1,95 \pm 0,87$ & $1,56 \pm 0,81$ \\
\hline $\mathrm{Cs}\left(\mathrm{mg} \mathrm{dm}^{-3}\right)$ & $62,0 \pm 12,8$ & $65,9 \pm 12,9$ & $101,9 \pm 84,3$ \\
\hline$P$ remanescente $\left(\mathrm{mg} \mathrm{dm}^{-3}\right)$ & $19,4 \pm 5,3$ & $16,7 \pm 5,3$ & $15,3 \pm 5,6$ \\
\hline $\mathrm{pH} \mathrm{H}_{2} \mathrm{O}$ & $5,08 \pm 0,19$ & $5,04 \pm 0,27$ & $5,07 \pm 0,23$ \\
\hline $\mathrm{P}\left(\mathrm{mg} \mathrm{dm}^{-3}\right)$ & $2,58 \pm 0,64$ & $2,29 \pm 0,35$ & $2,13 \pm 0,35$ \\
\hline $\mathrm{K}\left(\mathrm{mg} \mathrm{dm} \mathrm{m}^{-3}\right)$ & $40,2 \pm 22,0$ & $27,9 \pm 15,7$ & $24,3 \pm 17,5$ \\
\hline $\mathrm{Ca}+\mathrm{Mg}\left(\mathrm{cmol}_{\mathrm{c}} \mathrm{dm}^{-3}\right)$ & $0,34 \pm 0,32$ & $0,18 \pm 0,16$ & $0,19 \pm 0,15$ \\
\hline $\mathrm{Al}\left(\mathrm{cmol}_{\mathrm{c}} \mathrm{dm}^{-3}\right)$ & $0,54 \pm 0,35$ & $0,74 \pm 0,33$ & $0,77 \pm 0,38$ \\
\hline $\mathrm{H}+\mathrm{Al}\left(\mathrm{cmol}_{\mathrm{c}} \mathrm{dm}^{-3}\right)$ & $7,40 \pm 1,13$ & $7,69 \pm 1,03$ & $7,45 \pm 1,27$ \\
\hline $\operatorname{ADA}\left(\mathrm{kg} \mathrm{kg}^{-1}\right)$ & $0,21 \pm 0,04$ & $0,24 \pm 0,06$ & $0,26 \pm 0,06$ \\
\hline IEA $(\%)$ & $81,4 \pm 9,1$ & $85,3 \pm 6,6$ & $83,3 \pm 6,5$ \\
\hline Ds $\left(\mathrm{kg} \mathrm{dm}^{-3}\right)$ & $1,31 \pm 0,15$ & $1,27 \pm 0,12$ & $1,20 \pm 0,14$ \\
\hline Macroporosidade $\left(\mathrm{m} \mathrm{m}^{-3}\right)$ & $0,15 \pm 0,05$ & $0,17 \pm 0,05$ & $0,19 \pm 0,06$ \\
\hline Microporosidade $\left(\mathrm{m} \mathrm{m}^{-3}\right)$ & $0,36 \pm 0,05$ & $0,36 \pm 0,04$ & $0,36 \pm 0,06$ \\
\hline
\end{tabular}

$\mathrm{AM}$, atividade microbiana; CBM, carbono da biomassa microbiana; COT, carbono orgânico total; MOL, matéria orgânica leve em água; Cs, carbono solúvel em água; $\mathrm{H}+\mathrm{Al}$, acidez potencial; ADA, argila dispersa em água; IEA, índice de estabilidade de agregados; Ds, densidade do solo.

Pesq. agropec. bras., Brasília, v.51, n.9, p.1184-1193, set. 2016 DOI: 10.1590/S0100-204X2016000900018 
superficial ocasionada pelo intenso tráfego de animais, e pela matéria orgânica do solo que, em razão da maior atividade microbiana e do contínuo aporte de material vegetal, dá origem a uma matéria orgânica de composição diferente, em profundidade no solo (Moreira et al., 2005; Boeni et al., 2014).

O conjunto de variáveis com os atributos das três profundidades produziu uma função discriminante de maior exatidão. Assim, a avaliação dos níveis de degradação pôde ser simplificada a partir de atributos do solo com a função discriminante (Tabela 7). Entre os atributos, à profundidade de $0,00-0,05 \mathrm{~m}$, os teores de $\mathrm{Ca}+\mathrm{Mg}$ e $\mathrm{MOL}$ tiveram melhor participação na separação dos níveis de degradação, pois apresentaram as maiores cargas discriminantes.
Entre os dois níveis de degradação das pastagens, os maiores teores de $\mathrm{K}, \mathrm{P}$ e $\mathrm{Ca}+\mathrm{Mg}$ foram encontrados no nível baixo a moderado de degradação (Tabela 8), que apresentou maior percentual de cobertura por Urochloa. Schaefer et al. (2002) afirmaram que nutrientes como $\mathrm{K}, \mathrm{P}, \mathrm{Ca}$ e $\mathrm{Mg}$ podem ser perdidos em ambientes que não apresentam cobertura vegetal suficiente para exercer a proteção contra a erosão, conforme identificado no nível alto a muito alto de degradação.

Os teores de $\mathrm{Ca}+\mathrm{Mg}$ foram similares entre mata e nível alto a muito Alto de degradação, da mesma forma que os teores de $\mathrm{K}$ entre mata e nível baixo a moderado de degradação foram similares (Tabela 8). Assim, estudos de indicadores de qualidade do solo

Tabela 6. Resumo do procedimento de seleção de atributos ("forward stepwise"), para as três camadas de solo avaliadas, com um conjunto de dados pré-selecionados que uniu as três profundidades.

\begin{tabular}{|c|c|c|c|c|c|}
\hline \multirow{3}{*}{$\begin{array}{l}\text { Profundidade } \\
\text { (m) }\end{array}$} & \multirow[t]{3}{*}{ Variáveis selecionadas ${ }^{(1)}$} & \multicolumn{4}{|c|}{ Parâmetros das funções discriminantes } \\
\hline & & \multirow{2}{*}{$\begin{array}{l}\text { Lambda } \\
\text { de Wilk }\end{array}$} & \multirow[t]{2}{*}{ Valor $\mathrm{p}$} & \multicolumn{2}{|c|}{ Percentagem de acerto (\%) } \\
\hline & & & & Baixo a moderado & Alto a muito alto \\
\hline $0,00-0,05$ & $\mathrm{MOL}, \mathrm{H}+\mathrm{Al}, \mathrm{COT}, \mathrm{DS}, \mathrm{Ca}+\mathrm{Mg}$ & 0,242 & 0,0006 & 84 & 100 \\
\hline $0,05-0,10$ & $\mathrm{~K}, \mathrm{DS}, \mathrm{P}, \mathrm{COT}$ & 0,344 & 0,0029 & 75 & 87 \\
\hline $0,10-0,20$ & $\mathrm{~K}, \mathrm{DS}, \mathrm{COT}$ & 0,559 & 0,0228 & 58 & 100 \\
\hline Todas & $\mathrm{K}_{0,05-10 \mathrm{~m}}, \mathrm{MOL}_{0,05-0,10 \mathrm{~m}}, \mathrm{DS}_{0,05-0,10 \mathrm{~m}}, \mathrm{Ca}+\mathrm{Mg}_{0-0,05 \mathrm{~m}}, \mathrm{~K}_{0,10-0,20 \mathrm{~m}}, \mathrm{P}_{0,05-0,10 \mathrm{~m}}$ & 0,102 & $<0,0001$ & 100 & 100 \\
\hline
\end{tabular}

${ }^{(1)} \mathrm{COT}$, carbono orgânico total $\left(\mathrm{g} \mathrm{kg}^{-1}\right)$; MOL, matéria orgânica leve em água, $\mathrm{g} \mathrm{kg}^{-1} ; \mathrm{P}, \mathrm{mg} \mathrm{dm}^{-3} ; \mathrm{K} \mathrm{mg} \mathrm{dm}^{-3} ; \mathrm{Ca}+\mathrm{Mg} \mathrm{cmolc} \mathrm{dm}^{-3} ; \mathrm{H}+\mathrm{Al}$, acidez potencial, cmolc $\mathrm{dm}^{-3}$; Ds, densidade do solo, $\mathrm{kg} \mathrm{dm}^{-3}$.

Tabela 7. Seleção de variáveis "forward stepwise", coeficientes da função discriminante gerada com atributos das três profundidades, para a classificação da degradação das pastagens em níveis baixo a moderado, alto a muito alto ${ }^{(1)}$, além das cargas discriminantes.

\begin{tabular}{|c|c|c|c|c|c|c|}
\hline Passo & Entrada & Lambda de Wilk & Valor F & Valor $\mathrm{p}$ & $\begin{array}{l}\text { Coeficientes da função } \\
\text { discriminante }\end{array}$ & $\begin{array}{c}\text { Cargas } \\
\text { discriminantes }^{(3)}\end{array}$ \\
\hline 1 & $\mathrm{~K}_{0,05-0,10 \mathrm{~m}}$ & 0,57 & 13,40 & 0,0018 & $-5,805$ & 0,71 \\
\hline 2 & $\mathrm{MOL}_{0-0,05 \mathrm{~m}}$ & 0,44 & 4,70 & 0,0446 & 38,322 & $-0,69$ \\
\hline 3 & $\mathrm{DS}_{0,05-0,10 \mathrm{~m}}$ & 0,34 & 4,87 & 0,0424 & 0,381 & 0,42 \\
\hline 4 & $\mathrm{Ca}+\mathrm{Mg}_{0-0,05 \mathrm{~m}}$ & 0,25 & 5,18 & 0,0380 & 127,222 & 0,58 \\
\hline 5 & $\mathrm{~K}_{0,10-0,20 \mathrm{~m}}$ & 0,15 & 9,07 & 0,0093 & 13,700 & 0,63 \\
\hline 6 & $\mathrm{P}_{0,05-0,10 \mathrm{~m}}$ & 0,10 & 6,61 & 0,0233 & $-0,778$ & 0,48 \\
\hline Escore corte crítico ${ }^{(2)}$ & & & & & 176,59 & \\
\hline
\end{tabular}

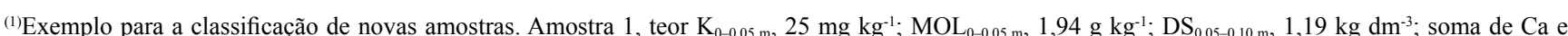
$\mathrm{Mg}_{0-0,05} \mathrm{~m}$, respeitando-se a relação próxima de 3:1, 0,70 $\mathrm{cmol}_{\mathrm{c}} \mathrm{dm}^{-3}$; teor $\mathrm{K}_{0,10-0,20 \mathrm{~m}}, 17 \mathrm{mg} \mathrm{kg}^{-1} ; \mathrm{P}_{0,05-0,10 \mathrm{~m}}, 2,58 \mathrm{mg} \mathrm{dm}^{-3}$ - escore calculado amostra $1=198,84$. Amostra 2, teor $\mathrm{K}_{0-0,05 \mathrm{~m}}, 11 \mathrm{mg} \mathrm{kg}^{-1} ; \mathrm{MOL}_{0-0,05 \mathrm{~m}}, 6,30 \mathrm{~g} \mathrm{~kg}^{-1} ; \mathrm{DS}_{0,05-0,10 \mathrm{~m}}, 1,25 \mathrm{~kg} \mathrm{dm}^{-3}$; soma de $\mathrm{Ca}$ e $\mathrm{Mg}_{0-0,05 \mathrm{~m}}$, respeitando-se a relação próxima de 3:1, 0,19 $\mathrm{cmol}_{\mathrm{c}} \mathrm{dm}^{-3}$; teor $\mathrm{K}_{0,10-0,20 \mathrm{~m}}, 10 \mathrm{mg} \mathrm{kg}^{-1} ; \mathrm{P}_{0,05-0,10 \mathrm{~m}}, 2,07 \mathrm{mg} \mathrm{dm}^{-3}$ - Escore calculado amostra $2=153,96$. Amostra 1 - escore calculado (198,84) maior que $\mathrm{o}$ escore crítico $(176,59)$, classificação de nível de degradação baixo a moderado. Amostra 2 - escore calculado $(153,84)$ menor que o escore crítico $(176,59)$, classificação de nível de degradação alto a muito alto. ${ }^{(2)}$ Escore de corte crítico para amostras com valor de n desiguais (Hair et al., 2005). ${ }^{(3)}$ Forte carga fatorial, >0,75. Moderada carga fatorial, 0,5 a 0,74 (Hair et al., 2005). 
devem ser cautelosos ao utilizar atributos de solo de matas nativas como referência de qualidade, uma vez que esses sistemas podem ter sido perturbados e podem ainda estar em fase de resiliência, como no caso do presente estudo.

Os maiores teores de MOL foram encontrados no nível alto a muito alto de degradação (Tabela 8). Araújo et al. (2011) também observaram maior quantidade de MOL, em solo sob mata e pastagem com elevada infestação de vegetação espontânea. Isso indica que nem sempre a maior oferta de forragem favorece a maior quantidade da fração leve da matéria orgânica, o que corrobora Lima et al. (2011), que não encontraram relação entre liteira depositada e matéria orgânica leve em pastagens de Urochloa brizantha. Esse fato é atribuído à maior relação $\mathrm{C} / \mathrm{N}$ em área de floresta, pois ocorre incremento do conteúdo de carbono em folhas, ramos e galhos, o que aumenta, além da relação $\mathrm{C} / \mathrm{N}$, a presença de compostos mais recalcitrantes como lignina (Araújo et al., 2011; Clemente et al., 2011). Assim, no presente estudo, a vegetação espontânea de folhas largas, com seus ramos e galhos, pode ter contribuído para a elevação do conteúdo de lignina da MOL e para sua maior concentração na camada superficial, em comparação ao nível baixo de degradação. Sugere-se, portanto, que estudos que visem quantificar a matéria orgânica, para distinção de níveis de degradação de pastagens, com vegetação de cobertura ou diversificada, também levem em consideração a composição química do material aportado ao solo.

Tabela 8. Média dos atributos do solo, conforme os níveis de degradação avaliados e na mata selecionada ${ }^{(1)}$.

\begin{tabular}{lccc}
\hline \multirow{2}{*}{ Atributos } & \multicolumn{2}{c}{ Nível de degradação } & Mata \\
\cline { 2 - 3 } & Alto a muito alto & Baixo a moderado & \\
\hline $\mathrm{K}_{0,05-0,10 \mathrm{~m}}$ & $15,60 \mathrm{~b}$ & $36,10 \mathrm{a}$ & $51,30 \mathrm{a}$ \\
$\mathrm{MOL}_{0,05-0,10 \mathrm{~m}}$ & $4,34 \mathrm{a}$ & $2,48 \mathrm{~b}$ & $2,05 \mathrm{~b}$ \\
$\mathrm{DS}_{0,05-0,10 \mathrm{~m}}$ & $1,31 \mathrm{a}$ & $1,21 \mathrm{a}$ & $1,20 \mathrm{a}$ \\
$\mathrm{Ca}+\mathrm{Mg}_{0,05-0,10 \mathrm{~m}}$ & $0,12 \mathrm{~b}$ & $0,48 \mathrm{a}$ & $0,21 \mathrm{~b}$ \\
$\mathrm{~K}_{0,10-0,20 \mathrm{~m}}$ & $15,70 \mathrm{~b}$ & $36,10 \mathrm{a}$ & $37,30 \mathrm{a}$ \\
$\mathrm{P}_{0,05-0,10 \mathrm{~m}}$ & $2,10 \mathrm{~b}$ & $2,42 \mathrm{a}$ & $2,79 \mathrm{a}$ \\
\hline $\mathrm{AM}_{0,05-0,10 \mathrm{~m}}$ & $41,9 \mathrm{~b}$ & $51,0 \mathrm{~b}$ & $68,56 \mathrm{a}$ \\
\hline
\end{tabular}

${ }^{(1)}$ Médias seguidas de letras iguais nas linhas, não diferem pelo teste de Tukey Post-Hoc, a 5\% de probabilidade. MOL, matéria orgânica leve em água, $\mathrm{g} \mathrm{kg}^{-1} ; \mathrm{P}, \mathrm{mg} \mathrm{dm}^{-3} ; \mathrm{K}, \mathrm{mg} \mathrm{dm}^{-3} ; \mathrm{Ca}+\mathrm{Mg}, \mathrm{cmol}_{\mathrm{c}} \mathrm{dm}^{-3} ; \mathrm{Ds}$, densidade do solo, $\mathrm{kg} \mathrm{dm}^{-3}$; e AM, atividade microbiana, $\mathrm{mg} \mathrm{C}-\mathrm{CO}_{2} 100 \mathrm{~cm}^{-3}$.
A atividade microbiana foi estatisticamente maior $(\mathrm{p}=0,012)$ em área de mata $\left(68,56 \mathrm{mgC}-\mathrm{CO}_{2} 100 \mathrm{~cm}^{-3}\right)$ do que no nível de degradação alto $\left(41,9 \mathrm{mg} \mathrm{C}-\mathrm{CO}_{2}\right.$ $100 \mathrm{~cm}^{-3}$ ). Melo et al. (2012) também observaram maiores taxas de emissão de $\mathrm{CO}_{2}$, em Latossolo sob floresta, do que em pastagem de Urochloa. Segundo os autores, isso pode ocorrer em consequência da contínua adição de resíduos vegetais diversificados e mais lábeis, que estimulam os microrganismos heterotróficos e a ciclagem de nutrientes. A densidade do solo não apresentou forte relação com os níveis de degradação, com significância entre níveis de degradação somente acima de $15 \%$ (Tabela 8 ).

\section{Conclusões}

1. Os indicadores de qualidade do solo estão relacionados aos níveis de degradação das pastagens e os mais sensíveis são a matéria orgânica leve e o conteúdo de $\mathrm{Ca}+\mathrm{Mg}$, à profundidade $0,00-0,05 \mathrm{~m}$, e o conteúdo de $\mathrm{K}$ às profundidades $0,05-0,10$ e $0,10-0,20 \mathrm{~m}$.

2. Os indicadores de qualidade de solo permitem separar como baixo a moderado e alto a muito alto os níveis de degradação de pastagens em Latossolo.

3. Na análise visual, a presença de solo exposto é a variável mais assertiva em discriminar os diferentes níveis de degradação.

\section{Agradecimentos}

Ao Conselho Nacional de Desenvolvimento Científico e Tecnológico (CNPq); à Fundação de Amparoà Pesquisa e Inovaçãodo EspíritoSanto(Fapes); à Universidade Federal do Espírito Santo (Ufes), Programa de Pós-Graduação em Produção Vegetal; e à Embrapa Solos, pelo apoio ao desenvolvimento do projeto; à Coordenação de Aperfeiçoamento de Pessoal de Nível Superior (Capes), pela concessão de bolsa; aos agricultores, por permitirem a coleta de informações em suas áreas.

\section{Referências}

ALBERNAZ, W.M.; LIMA, J.M. de. Caracterização da cobertura vegetal de pastagens em duas sub-bacias hidrográficas da região de Lavras, MG. Ciência e Agrotecnologia, v.31, p.290-297, 2007. DOI: $10.1590 / \mathrm{S} 1413-70542007000200004$.

ALVAREZ V., V.H.; NOVAIS, R.F.; DIAS, L.E.; OLIVEIRA, J.A. Determinação e uso do fósforo remanescente. Boletim Informativo da Sociedade Brasileira de Ciência do Solo, v.25, p.27-32, 2000. 
ANDRADE, R.G.; RODRIGUES, C.A.G.; SANCHES, I.D'A.; TORRESAN, F.E.; QUARTAROLI, C.F. Uso de técnicas de sensoriamento remoto na detecção de processos de degradação de pastagens. Engenharia na Agricultura, v.21, p.234-243, 2013. DOI: 10.13083/1414-3984.v21n03a02.

ARAÚJO, E.A. de; KER, J.C.; MENDONÇA, E. de S.; SILVA, I.R. da; OLIVEIRA, E.K. Impacto da conversão floresta-pastagem nos estoques e na dinâmica do carbono e substâncias húmicas no solo do bioma Amazônico. Acta Amazonica, v.41, p.103-114, 2011. DOI: $10.1590 /$ S0044-59672011010200001.

ARAUJO-JUNIOR, C.F.; GUIMARÃES, P.T.G.; DIAS JUNIOR, M. de S.; ALCÂNTARA, E.N.; MENDES, A.D.R. Alterações nos atributos químicos de um Latossolo pelo manejo de plantas invasoras em cafeeiros. Revista Brasileira de Ciência do Solo, v.35, p.22072217, 2011. DOI: 10.1590/S0100-06832011000600036.

BARRETO, P.; SARTORI, M. Levantamento de áreas agrícolas degradadas no Estado do Espírito Santo. Vitória: Cedagro, 2012.

BOENI, M.; BAYER, C.; DIECKOW, J.; CONCEIÇÃO, P.C.; DICK, D.P.; KNICKER, H.; SALTON, J.C.; MACEDO, M.C.M. Organic matter composition in density fractions of Cerrado Ferralsols as revealed by CPMAS ${ }^{13} \mathrm{C}$ NMR: influence of pastureland, cropland and integrated crop-livestock. Agriculture, Ecosystems and Environment, v.190, p.80-86, 2014. DOI: 10.1016/j.agee.2013.09.024.

CLEMENTE, J.S.; SIMPSON, A.J.; SIMPSON, M.J. Association of specific organic matter compounds in size fractions of soils under different environmental controls. Organic Geochemistry, v.42, p.1169-1180, 2011. DOI: 10.1016/j.orggeochem.2011.08.010.

COSTA, K.A. de P.; ROSA, B.; OLIVEIRA, I.P. de; CUSTÓDIO, D.P.; SILVA, D.C. e. Efeito da estacionalidade na produção de matéria seca e composição bromatológica da Brachiaria brizantha cv. Marandu. Ciência Animal Brasileira, v.6, p.187-193, 2005.

COSTA, M.A.T.; TORMENA, C.A.; LUGÃO, S.M.B.; FIDALSKI, J.; NASCIMENTO, W.G.; MEDEIROS, F.M. Resistência do solo à penetração e produção de raízes e de forragem em diferentes níveis de intensificação do pastejo. Revista Brasileira de Ciência do Solo, v.36, p.993-1004, 2012. DOI: 10.1590/S010006832012000300029.

COSTA, O.V.; COSTA, L.M.; FONTES, L.E.F.; ARAUJO, Q.R.; KER, J.C.; NACIF, P.G.S. Cobertura do solo e degradação de pastagens em área de domínio de Chernossolos no sul da Bahia. Revista Brasileira de Ciência do Solo, v.24, p.843-856, 2000. DOI: 10.1590/S0100-06832000000400016.

DONAGEMA, G.K.; CAMPOS, D.V.B de; CALDERANO, S.B.; TEIXEIRA, W.G.; VIANA, J.H.M. (Org.). Manual de métodos de análise de solo. 2.ed. rev. Rio de Janeiro: Embrapa Solos, 2011. 230p. (Embrapa Solos. Documentos, 132).

HAIR JR., J.F.; ANDERSON, R.E.; TATHAM, R.L.; BLACK, W.C. Análise multivariada de dados. 5.ed. Porto Alegre: Bookman, 2005. 593p.

INCAPER. INSTITUTO CAPIXABA DE PESQUISA, ASSISTÊNCIA TÉCNICA E EXTENSÃO RURAL. Série Histórica: dados médios da série histórica da estação meteorológica localizada no Município de Alegre-ES. Vitória:
Incaper, 2014. Disponível em: <http://hidrometeorologia.incaper. es.gov.br/?pagina=alegre_sh $>$. Acesso em: 31 jan. 2014.

LACERDA, N.B.; ZERO, V.M.; BARILLI, J.; MORAES, M.H.; BICUDO, S.J. Efeito de sistemas de manejo na estabilidade de agregados de um Nitossolo Vermelho. Engenharia Agrícola, v.25, p.686-695, 2005. DOI: 10.1590/S0100-69162005000300014.

LIMA, S.S. de; ALVES, B.J.R.; AQUINO, A.M. de; MERCANTE, F.M.; PINHEIRO, E.F.M.; SANT'ANNA, S.A.C.; URQUIAGA, S.; BODDEY, R.M. Relação entre a presença de cupinzeiros e a degradação de pastagens. Pesquisa Agropecuária Brasileira, v.46, p.1699-1706, 2011. DOI: 10.1590/S0100-204X2011001200016.

MARCHÃO, R.L.; BALBINO, L.C.; SILVA, E.M. da; SANTOS JUNIOR, J. de D.G.; SÁ, M.A.C. de; VILELA, L.; BECQUER, T. Qualidade física de um Latossolo Vermelho sob sistemas de integração lavoura-pecuária no Cerrado. Pesquisa Agropecuária Brasileira, v.42, p.873-882, 2007. DOI: 10.1590/S0100$204 X 2007000600015$.

MCINNES, W.S.; SMITH, B.; MCDERMID, G.J. Discriminating native and nonnative grasses in the dry mixedgrass prairie with MODIS NDVI time series. IEEE Journal of Selected Topics in Applied Earth Observations and Remote Sensing, v.8, p.13951403, 2015. DOI: 10.1109/JSTARS.2015.2416713.

MELLONI, R.; MELLONI, E.G.P.; ALVARENGA, M.I.N.; VIEIRA, F.B.M. Avaliação da qualidade de solos sob diferentes coberturas florestais e de pastagem no sul de Minas Gerais. Revista Brasileira de Ciência do Solo, v.32, p.2461-2470, 2008. DOI: 10.1590/S0100-06832008000600023.

MELO, V.S.; DESJARDINS, T.; SILVA JR, M.L.; SANTOS, E.R.; SARRAZIN, M.; SANTOS, M.M.L.S. Consequences of forest conversion to pasture and fallow on soil microbial biomass and activity in the eastern Amazon. Soil Use and Management, v.28, p.530-535, 2012. DOI: 10.1111/sum.12003.

MENDONÇA, E. de S.; MATOS, E. da S. (Ed.). Matéria orgânica do solo: métodos de análises. Viçosa: UFV, 2005. 81p.

MICHELON, C.J.; CARLESSO, R.; PETRY, M.T.; MELO, G.L.; SPOHR, R.B.; ANDRADE, J.G. de. Qualidade física dos solos irrigados de algumas regiões do Brasil central. Revista Brasileira de Engenharia Agrícola e Ambiental, Campina Grande, v.13, p.39-45, 2009. DOI: 10.1590/s1415-43662009000100006.

MOREIRA, J.A.A.; OLIVEIRA, I.P. de; GUIMARÃES, C.M.; STONE, L.F. Atributos químicos e físicos de um Latossolo Vermelho distrófico sob pastagens recuperada e degradada. Pesquisa Agropecuária Tropical, v.35, p.155-161, 2005.

MURPHY, C.A.; FOSTER, B.L.; RAMSPOTT, M.E.; PRICE, K.P. Effects of cultivation history and current grassland management on soil quality in northeastern Kansas. Journal of Soil and Water Conservation, v.61, p.75-89, 2006.

NIERO, L.A.C.; DECHEN, S.C.F.; COELHO, R.M.; DE MARIA, I.C. Avaliações visuais como índice de qualidade do solo e sua validação por análises físicas e químicas em um Latossolo Vermelho distroférrico com usos e manejos distintos. Revista Brasileira de Ciência do Solo, v.34, p.1271-1282, 2010. DOI: 10.1590/S010006832010000400025.

PESSOA, P.M. de A.; DUDA, G.P.; BARROS, R.B. de; FREIRE, M.B.G. dos S.; NASCIMENTO, C.W.A. do; CORREA, M.M. 
Frações de carbono orgânico de um Latossolo húmico sob diferentes usos no agreste brasileiro. Revista Brasileira de Ciência do Solo, v.36, p.97-104, 2012. DOI: 10.1590/S010006832012000100011.

PREZOTTI, L.C.; GOMES, J.A.; DADALTO, G.G.; OLIVEIRA, J.A. de. Manual de recomendação de calagem e adubação para o Estado do Espírito Santo: $5^{\text {a }}$ aproximação. Vitória: Seea/ Incaper/Cedagro, 2007. 305p.

ROCHA JUNIOR, P.R. da; DONAGEMMA, G.K.; ANDRADE, F.V.; PASSOS, R.R.; BALIEIRO, F. de C.; MENDONÇA, E. de S.; RUIZ, H.A. Can soil organic carbon pools indicate the degradation levels of pastures in the Atlantic Forest biome? Journal of Agricultural Science, v.6, p.84-95, 2014. DOI: 10.5539/jas. v6n1p84.

SANTOS, A.C. dos; FERREIRA, E.M.; ARAÚJO, L.C. de. Propriedades químicas e físicas de solos em áreas sob pastagens em cerrado do norte do Tocantins. Revista Acadêmica: Ciência Animal, v.7, p.55-63, 2009a.

SANTOS, H.G. dos; JACOMINE, P.K.T.; ANJOS, L.H.C. dos; OLIVEIRA, V.A. de; LUMBRERAS, J.F.; COELHO, M.R.; ALMEIDA, J.A. de; CUNHA, T.J.F.; OLIVEIRA, J.B. de. Sistema brasileiro de classificação de solos. 3.ed. rev. e ampl. Brasília: Embrapa, 2013. 353p.
SANTOS, L.N.S. dos; PASSOS, R.R.; CARDOSO, L.C.M.; SANTOS, C.L.; GARCIA, G. de O.; CECÍLIO, R.A. Avaliação de atributos físicos de um Latossolo sob diferentes coberturas vegetais em Alegre (ES). Engenharia Ambiental, v.6, p.140149, 2009b.

SCHAEFER, C.E.R.; SILVA, D.D.; PAIVA, K.W.N.; PRUSKI, F.F.; ALBUQUERQUE FILHO, M.R.; ALBUQUERQUE, M.A. Perdas de solo, nutrientes, matéria orgânica e efeitos microestruturais em Argissolo Vermelho-Amarelo sob chuva simulada. Pesquisa Agropecuária Brasileira, v.37, p.669-678, 2002. DOI: 10.1590/ S0100-204X2002000500012.

SHAPIRO, S.S.; WILK, M.B. An analysis of variance test for normality (complete samples). Biometrika, v.52, p.591-611, 1965. DOI: $10.1093 /$ biomet/52.3-4.591.

SPAIN, J.M.; GUALDRÓN, R. Degradación y rehabilitación de pasturas. In: LASCANO, C.E.; SPAIN, J.M. (Ed.). Establecimiento y renovación de pasturas: conceptos, experiencias y enfoque de la investigación. Cali: CIAT, 1988. p.269-283.

TRASAR-CEPEDA, C.; LEIRÓS, C.; GIL-SOTRES, F.; SEOANE, S. Towards a biochemical quality index for soils: An expression relating several biological and biochemical properties. Biology and Fertility of Soils, v.26, p.100-106, 1998. DOI: $10.1007 / \mathrm{s} 003740050350$.

Recebido em 31 de agosto de 2015 e aprovado em 22 de dezembro de 2015 